\title{
Does trastuzumab-related cardiotoxicity influence long-term outcome in patients with HER-2 positive breast cancer? A prospective study
}

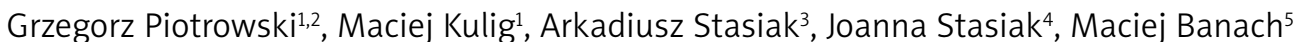

\author{
${ }^{1}$ Cardiology Department, Nicolaus Copernicus Memorial Hospital, Lodz, Poland \\ 2Department of Cardiooncology, Medical University, Lodz \\ ${ }^{3}$ Department of Physiology, Development and Neuroscience, University of Cambridge, \\ Cambridge, UK \\ ${ }^{4}$ Department of Chemical Engineering and Biotechnology, University of Cambridge, \\ Cambridge, UK \\ ${ }^{5}$ Department of Hypertension, Medical University of Lodz, Lodz, Poland
}

Submitted: 29 April 2020

Accepted: 25 May 2020

Arch Med Sci

DOI: https://doi.org/10.5114/aoms/122841

Copyright @ 2021 Termedia \& Banach

\begin{abstract}
Introduction: Trastuzumab is a monoclonal antibody directed against the HER-2 receptor that has led, in an adjuvant setting, to higher disease-free (DFS) and overall survival (OS) in HER-2 positive breast cancer (BC) compared with chemotherapy alone. Cardiotoxicity often results in early discontinuation of trastuzumab, which may elevate the risk of cancer recurrence or mortality. Our study aimed to assess how early interruption or early permanent termination of adjuvant trastuzumab treatment influences DFS and OS of patients with HER-2 positive BC.
\end{abstract}

Material and methods: This is a prospective observation of 253 women ( $55 \pm 10$ years of age) with HER-2 positive unilateral, non-metastatic BC treated with trastuzumab in an adjuvant setting. To monitor the safety of the treatment echocardiography was performed at baseline and every 3 months up to 12 months after the end of therapy. If cardiotoxicity developed, trastuzumab was stopped early. Overall survival and DFS were assessed.

Results: Trastuzumab-associated cardiac complications resulting in treatment discontinuation developed in 52 (20.55\%) patients. Median DFS time was 21.1 months in the group with interruption compared with 25.7 months in the group with full trastuzumab treatment, being significantly shorter $(\mathrm{HR}=2.32,95 \% \mathrm{Cl}: 1.15-4.71, p=0.0106)$. Two year OS in the interruption and no-interruption groups were $80.8 \%$ and $88.5 \%$, respectively, which were not statistically significantly different $(p=0.268)$. In a multivariate regression analysis the cumulative dose of anthracycline $(\mathrm{OR}=1.01,95 \% \mathrm{Cl}$ : $1.00-1.01, p=0.002)$ and LVEF at baseline $(\mathrm{OR}=0.83,95 \% \mathrm{Cl}: 0.70-0.99$, $p=0.0344$ ) were independent predictors of a cardiotoxic effect.

Conclusions: Trastuzumab-related cardiotoxicity resulting in early treatment discontinuation negatively influences DFS, but does not seem to influence OS.

Key words: cardiotoxicity, disease-free survival, overall survival, breast cancer adjuvant treatment, non-metastatic breast cancer, breast cancer prognosis.

\section{Introduction}

Approximately $20-25 \%$ of breast cancers overexpress the human epidermal growth factor receptor 2 (HER-2), which is associated with a very

\author{
Corresponding author: \\ Grzegorz Piotrowski \\ Department of Cardiology \\ Nicolaus Copernicus \\ Memorial Hospital \\ 62 Pabianicka St. \\ 91-513 Łódź, Poland \\ E-mail: gppiotr4@wp.pl
}


bad prognosis [1, 2]. Trastuzumab is a humanized monoclonal antibody directed against the HER-2 receptor that has led to higher disease-free (DFS) and overall survival (OS) in HER-2 positive breast cancer as compared with chemotherapy alone. Trastuzumab is usually well tolerated, but, because of cardiotoxicity, mainly asymptomatic left ventricle dysfunction and less frequently overt heart failure (HF), it is often interrupted or terminated early. In pivotal adjuvant trials with trastuzumab treatment the symptomatic HF ranged from 0.1 to $5.1 \%$ and an asymptomatic left ventricle ejection fraction (LVEF) drop was observed in $3.2-19 \%$ of patients [3-11]. However, in everyday practice the frequency of trastuzumab related cardiotoxicity is usually much higher. Cardiotoxicity is the main limitation in the use of trastuzumab. Since standard of care adjuvant trastuzumab therapy in breast cancer lasts for 12 months, early discontinuation may elevate cancer recurrence rates due to inadequate treatment. A reduced dose of trastuzumab may also have a negative impact on OS. In a meta-analysis of randomized clinical trials trastuzumab treatments shorter than 12 months resulted in poorer cancer-free and overall survival [3]. There is evidence that, regardless of the optimal duration of trastuzumab therapy, interruptions of the treatment may be associated with a poorer outcome. On the other hand, longterm reversibility of trastuzumab related cardiotoxicity remains controversial. There are some retrospective observations suggesting that asymptomatic LV dysfunction may not be as benign and reversible as it had been previously regarded [12-14]. A meta-analysis of randomised trials with trastuzumab for early-stage HER-2 positive breast cancer with a median follow-up of 36 months revealed that heart failure developed in $2.5 \%$ of patients [4]. This is why clinical guidelines recommend withholding trastuzumab if LVEF drops. Nevertheless, these recommendations do not result from evidence-based data. It is important to find a balance between the optimal trastuzumab treatment duration and cardiac short-term as well as long-term safety [15].

The purpose of the present study was to assess how early interruption or early permanent termination of adjuvant trastuzumab treatment influences DFS and OS in patients with HER-2 positive unilateral nonmetastatic breast cancer.

\section{Material and methods}

\section{Study design}

This is a prospective observation of 253 women with HER-2 positive unilateral, non-metastatic breast cancer treated with trastuzumab in an adjuvant setting from January $1^{\text {st }} 2011$ to December $31^{\text {st }} 2015$ in the Regional Centre for Oncology and Traumatology (Lodz, Poland), who were monitored for cardiac safety in the Cardiology Department.

\section{Population, treatment and methods}

The inclusion criteria followed clinical guidelines $[16,17]$ and were: histologically confirmed invasive HER-2 positive breast cancer and LVEF $\geq 50 \%$. The patients were excluded if they had metastatic disease at baseline, symptoms of heart failure, LVEF < $50 \%$ prior to initiation of trastuzumab, had myocardial infarction in the previous 6 months, or presented uncontrolled symptomatic angina pectoris, uncontrolled arrhythmia, uncontrolled hypertension or any significant valvular heart disease (mitral/aortic insufficiency/ stenosis). Each participant of the study signed informed consent. The study protocol was approved by the Ethics Committee of the Medical University of Lodz, Poland (number of the permission: RNN/107/08/KB). The study protocol conforms to the ethical guidelines of the 1975 Declaration of Helsinki.

HER-2 status was determined by immunohistochemical staining (3+), or in the case of HER-2 result 2++ amplification of the HER2 gene was evaluated using the fluorescence in situ hybridization (FISH) method.

The administered loading dose of trastuzumab was $8 \mathrm{mg} / \mathrm{kg}$ of body weight, and the maintenance dose was $6 \mathrm{mg} / \mathrm{kg}$ every three weeks for a total of 52 weeks. All breast cancer patients received anthracycline-based therapy prior to trastuzumab. The anthracycline-containing regimens used as adjuvant were AC (doxorubicin $60 \mathrm{mg} / \mathrm{m}^{2}$ and cyclophosphamide $600 \mathrm{mg} / \mathrm{m}^{2}$, every 3 weeks for four cycles) or FEC (fluorouracil $500 \mathrm{mg} / \mathrm{m}^{2}$, epirubicin $100 \mathrm{mg} / \mathrm{m}^{2}$, and cyclophosphamide $600 \mathrm{mg} /{ }^{2}$, every 3 weeks for 6 cycles). Docetaxel was given at a dose of $60-75 \mathrm{mg} / \mathrm{m}^{2}$ every three weeks. Adjuvant endocrine therapy and radiotherapy were applied when appropriate.

\section{Cardiotoxicity}

Cardiotoxicity was assumed as significant when the trastuzumab treatment was interrupted or prematurely discontinued. Cardiotoxicity was defined as occurrence of one of the following: (1) each absolute decrease of LVEF by $>15 \%$, (2) absolute reduction in LVEF by $10 \%$ from the baseline value and below the level of $50 \%$, (3) any symptoms or signs of heart failure $[16,17]$. Patients received further observation if they developed other, rare forms of cardiotoxicity (ECG abnormalities, conduction disorders) that were not defined at baseline and occurred during the treatment and were the reason for early tras- 
tuzumab discontinuation. In cases of significant cardiotoxicity trastuzumab was stopped early, before the completion of a full trastuzumab course. The decision regarding discontinuation of trastuzumab was made by the oncologist responsible for the treatment in cooperation with the supervising cardiologist for each patient individually, according to the guidelines $[9,16,17]$. When trastuzumab was discontinued HF treatment with angiotensin converting enzyme inhibitors/angiotensin receptor blockers (ACE-I/ARB) and/or $\beta$-blockers was initiated and up-titrated to the maximum tolerated doses as soon as possible. Additional cardiac treatment, including diuretics, anticoagulants, and antiarrhythmic drugs, was given as required by the clinical situation, based on published guidelines [16-18]. If LVEF recovered and rose at least up to $50 \%$ or over, trastuzumab was reinitiated. A patient was permanently excluded from trastuzumab treatment if they experienced a second significant drop of LVEF, which signified meeting the criteria of significant cardiotoxicity.

Any interruption of trastuzumab treatment for longer than 4 weeks was assumed significant and was included in the assessment and analysis.

\section{Echocardiography}

Echocardiography was performed and interpreted by the same experienced cardiologist (GP) at baseline and every 3 months during treatment up to 12 months after the end of the therapy. If LVEF dropped and met the criteria of cardiotoxicity, echocardiography was repeated every week to identify recovery of LV systolic function. All real-time two-dimensional transthoracic echocardiographic (TTE) examinations were performed using an i33 Philips ultrasound device with an S5-1 probe (United Kingdom, production year: 2010 and a 1.7-4.0 MHz transducer). Quantitative echocardiography was performed according to the current joint European Association of Echocardiography and American Society of Echocardiography guidelines [19]. Images were obtained in the parasternal long and short axis as well as apical views, with the subject lying in the left lateral position. Left ventricle ejection fraction (LVEF), LV end-diastolic (LVEDV) and LV end-systolic (LVESV) volumes were calculated using the Simpson biplane method [17, 19].

The following cardiovascular risk factors were analysed: age, body mass index (BMI), hypertension, diabetes mellitus, smoking, sedentary lifestyle, obesity, depression, positive family history and coronary artery disease. The following other (non-cardiovascular) risk factors of cardiotoxicity were analysed: cumulative dose of doxorubicin, treatment with cyclophosphamide, docetaxel, adjuvant radiotherapy, hormone therapy, and time from anthracycline termination to trastuzumab initiation [20-22, 24].

\section{Study outcomes}

The primary outcome of interest was to determine if cardiotoxicity-related early trastuzumab interruption/early termination affects DFS or OS in $B C$ patients treated in an adjuvant setting.

The secondary outcomes of interest were: 1) to determine the incidence of cardiotoxicity during adjuvant trastuzumab treatment; 2) to determine if there are any cardiovascular or chemotherapy related risk factors predisposing to trastuzumab cardiotoxicity.

Overall survival time was defined as the time from the diagnosis of cancer to death from any cause or the end of observation.

Disease-free survival was defined as the time from the diagnosis of cancer to recurrence of any type of BC. Relapse of cancer was assumed when a patient received palliative chemotherapy.

\section{Statistical analysis}

Data were reported as mean \pm standard deviation (SD). Comparisons between groups were performed with the unpaired Student's $t$-test for continuous variables and the $\chi^{2}$ test or Fisher's exact test as appropriate for categorical variables.

The differences in unadjusted all-cause mortality according to the complete trastuzumab treatment vs. early discontinuation of trastuzumab were assessed using Kaplan-Meier analysis and the log-rank test.

Univariate regression analysis was used to identify covariates of cardiotoxicity.

Logistic regression was used to assess the relationship between dependent variables and one of the independent variables. Then, all independent variables were included in a multivariate stepwise regression model to determine the best statistical predictor of the dependent variables. Statistical analysis software (SPSS and Statistica) were used to perform the analysis. A p-value less than 0.05 was considered statistically significant.

\section{Results}

The prospective observation included 253 consecutive women with HER-2 positive breast cancer who were treated with trastuzumab in an adjuvant setting and were monitored for cardiac safety in our Cardiology Department. The mean age of the study population was $55 \pm 10$ years of age (median 55 years old); mean baseline (before initiation of trastuzumab) LVEF $=60.66 \pm 4.88 \%$ (median $60.26 \%$ ). Other clinical features that characterise the study populations are presented in Tables I and II. There were no differences between study 
Table I. Cardiovascular risk factors at baseline in Group 1 (with significant trastuzumab-related cardiotoxicity) and in Group 2 (without significant trastuzumab-related cardiotoxicity)

\begin{tabular}{|lccc|}
\hline Risk factors & $\begin{array}{c}\text { Group 1 } \\
n=52\end{array}$ & $\begin{array}{c}\text { Group 2 } \\
n=201\end{array}$ & $\begin{array}{c}P \text {-value } \\
\text { Group 1 vs. Group 2 }\end{array}$ \\
\hline Age [years] & $56.8 \pm 9.6$ & $54.8 \pm 9.7$ & 0.366 \\
\hline BMI $\left[\mathrm{kg} / \mathrm{m}^{2}\right]$ & $27.3 \pm 4.4$ & $26.9 \pm 4.6$ & 0.417 \\
\hline Hypertension, $n$ (\%) & $22(47.8)$ & $77(38.3)$ & 0.327 \\
\hline Diabetes mellitus, $n(\%)$ & $3(6.5)$ & $13(6.5)$ & 0.952 \\
\hline Smoking, $n$ (\%) & $7(15.2)$ & $27(13.4)$ & 0.841 \\
\hline Hypercholesterolaemia, $n(\%)$ & $17(37.0)$ & $70(34.8)$ & 0.931 \\
\hline Sedentary life style, $n(\%)$ & $43(93.5)$ & $151(75.1)$ & 0.109 \\
\hline Overweight ( $\leq 25$ BMI $<30)$ & $19(41.3)$ & $80(39.8)$ & 0.864 \\
\hline Obesity (BMI $\geq 30)$ & $13(28.3)$ & $46(22.9)$ & 0.410 \\
\hline Depression, $n$ (\%) & $7(15.2)$ & $21(10.5)$ & 0.523 \\
\hline Positive family history, $n(\%)$ & $20(43.5)$ & $68(33.8)$ & 0.321 \\
\hline ACE-l/ARB (at baseline), $n(\%)$ & $16(34.8)$ & $62(30.9)$ & 0.172 \\
\hline$\beta$-blockers (at baseline), $n(\%)$ & $4(8.7)$ & $18(9.0)$ & 0.649 \\
\hline Coronary artery disease, $n(\%)$ & $0(0)$ & $10(5)$ & 1.000 \\
\hline
\end{tabular}

$B M I$ - body mass index, $A C E-I$ - angiotensin converting enzyme inhibitor, ARB - angiotensin II receptor blockers.

Table II. Non-cardiovascular risk factors for trastuzumab-related cardiotoxicity at baseline in Group 1 (with significant trastuzumab-related cardiotoxicity) and in Group 2 (without significant trastuzumab-related cardiotoxicity)

\begin{tabular}{|c|c|c|c|}
\hline Non-cardiovascular risk factor & $\begin{array}{c}\text { Group } 1 \\
n=52\end{array}$ & $\begin{array}{l}\text { Group } 2 \\
n=201\end{array}$ & $\begin{array}{c}\text { Group } 1 \\
\text { vs. Group } 2\end{array}$ \\
\hline $\mathrm{BSA}\left[\mathrm{m}^{2}\right]$ & $1.75 \pm 0.16$ & $1.74 \pm 0.15$ & 0.852 \\
\hline $\mathrm{BMI}\left[\mathrm{kg} / \mathrm{m}^{2}\right]$ & $27.90 \pm 5.20$ & $26.89 \pm 4.55$ & 0.459 \\
\hline Doxorubicin dose (mean) $\left[\mathrm{mg} / \mathrm{m}^{2}\right]$ & $358.58 \pm 80.88$ & $300.07 \pm 93.73$ & $<0.001$ \\
\hline Docetaxel, (n (\%)/dose [mg]) & $22(42.3 \%) / 507.07 \pm 443$ & $80(39.8 \%) / 469 \pm 231$ & $0.859 / 0.714$ \\
\hline Cyclophosphamide (n (\%)/dose [mg]) & $41(78.9 \%) / 3705.45 \pm 1027$ & $169(84.1 \%) / 2971.25 \pm 1796$ & $0.950 / 0.283$ \\
\hline 5-fluorouracyl (n (\%)/dose [mg]) & $14(26.9 \%) / 3282.05 \pm 1139$ & $39(19.4 \%) / 3628 \pm 3443$ & $0.203 / 0.06$ \\
\hline Adjuvant radiotherapy, $n(\%)$ & $33(63.5 \%)$ & $126(62.7 \%)$ & 0.440 \\
\hline Hormone therapy, $n$ (\%) & $12(23.1 \%)$ & $66(32.8 \%)$ & 0.393 \\
\hline Time anthracycline-trastuzumab [days] & $81.37 \pm 59.51(58)$ & $104.58 \pm 66.70(94)$ & 0.024 \\
\hline Left/right breast, $n$ & $27 / 22$ & $109 / 94$ & 0.736 \\
\hline
\end{tabular}

$B S A$ - body surface area, BMI - body mass index.

groups in terms of cardiovascular parameters (see Table I). In terms of other clinical parameters (non-cardiovascular risk factors for cardiotoxicity), patients who developed a significant cardiotoxic effect (Group 1) received a higher total doxorubicin dose ( $358.58 \pm 80.88$ vs. $300.07 \pm 93.73 \mathrm{mg} / \mathrm{m}^{2}$, $p<0.001)$ and had significantly shorter time from the completion of chemotherapy to the initiation of trastuzumab (81.37 \pm 59.51 vs. $104.58 \pm 66.70$ days; $p=0.024$ ) (see Table II). Neoadjuvant treatment that did not contain trastuzumab was ap- plied in 70 (27.7\%) patients. Radiotherapy prior to trastuzumab was performed in 159 patients (62.8\%). All patients underwent mastectomy; in 31 (12.26\%) women it was breast-conserving therapy $(\mathrm{BCT})$.

Fifty-two (20.55\%) patients (Group 1) had trastuzumab-associated cardiac complications resulting in discontinuation of the treatment. These included asymptomatic left ventricle dysfunction in 43 patients (16.99\%); symptomatic heart failure (NYHA III/IV) in 6 patients (2.37\%); new asymp- 


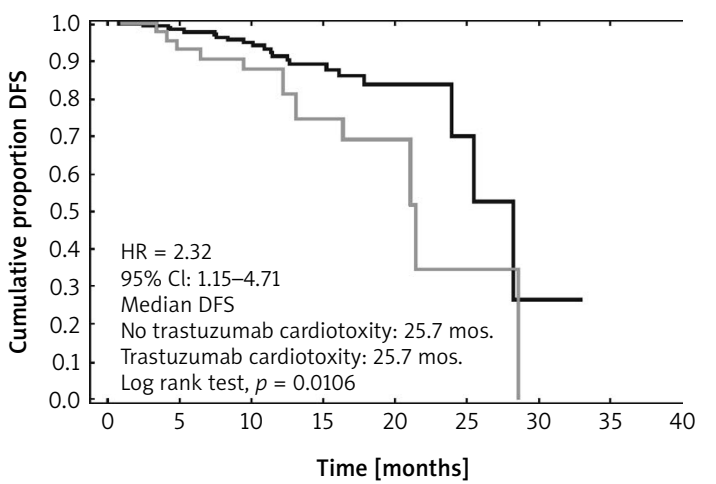

- No trastuzumab cardiotoxity

Trastuzumab cardiotoxity

Figure 1. Kaplan-Meier curves showing disease-free survival (DFS) for no trastuzumab cardiotoxicity vs. trastuzumab cardiotoxicity

tomatic left bundle branch block (LBBB) in $1 \mathrm{pa}$ tient $(0.39 \%)$; and new negative T-waves in ECG in 2 patients $(0.79 \%)$. The administration of trastuzumab was interrupted early in all these patients.

Trastuzumab treatment was stopped in 13 women after the first 3 months (in 1 due to asymptomatic LBBB); in 22 women after 6 months (in 2 due to ST-T changes in ECG and in 2 due to symptomatic HF); in 12 women after 9 months (in 1 due to symptomatic HF); and in 5 women after 12 months.

In 23 (48.1\%) patients LVEF improved and rose to the level of $50 \%$ or higher. In all cases LVEF recovery occurred after 4 weeks or longer. Mean time of LVEF recovery was $11 \pm 7$ weeks (median 9 weeks, 4-28 weeks). Trastuzumab was restarted only in $13(25 \%)$ patients, but in 6 (11.54\%) patients LVEF dropped again before the completion of the full trastuzumab course and the treatment was permanently terminated for them.

Guideline recommended HF treatment (angiotensin converting enzyme inhibitor, ACE-I/ $\beta$-blocker) was initiated in 35 (76.1\%) patients out of 46 with a significant LVEF drop. Twenty-two (47.8\%) women received both ACE-I and $\beta$-blocker; $8(17.4 \%)$ were on $\beta$-blockers; 5 (10.9\%) were only on ACE-I. Nine patients (19.6\%) were treated with a loop diuretic and 9 (19.6\%) with a mineralocorticoid receptor antagonist (1 with eplerenone, and the others with spironolactone).

Disease recurrence was observed in 33 patients (13\% of the total study population). Twenty-seven patients experienced relapse of cancer during adjuvant trastuzumab treatment. Thirteen of 33 patients with cancer recurrence had a significant cardiotoxic effect which was the cause of trastuzumab discontinuation, while 20 others did not.

Median DFS time was 21.1 months in the group with trastuzumab interruption (due to significant cardiotoxicity) (Group 1) and 25.7 months in the group with full trastuzumab treatment (and

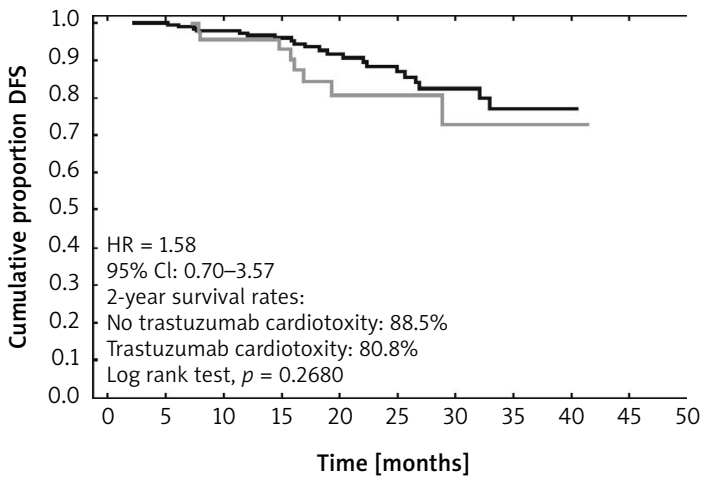

— No trastuzumab cardiotoxity _ Trastuzumab cardiotoxity

Figure 2. Kaplan-Meier curves showing overall survival (OS) for no trastuzumab cardiotoxicity vs. trastuzumab cardiotoxicity

with no significant cardiotoxicity) (Group 2). DFS was significantly shorter in the group that had a cardiotoxic effect (Group 1) (hazard ratio 2.32; 95\% Cl: 1.15-4.71, $p=0.0106)$.

Two-year overall survival (OS) rates in Group 1 and Group 2 were $80.8 \%$ and $88.5 \%$, respectively. Twenty-nine (11.5\%) patients died during the observation. In all cases death was assumed to be associated with cancer. Eight of the patients who died experienced significant cardiotoxicity, while 21 others did not. Cardiotoxicity-related early termination of trastuzumab did not affect OS negatively $(p=0.268)$. Median trastuzumab treatment time in Group 1 was 26.9 weeks.

The univariate analysis showed that none of the cardiovascular risk factors was significantly associated with a significant cardiotoxic effect. However, a significant association with cardiotoxic effect was observed for a cumulative dose of anthracycline $(\mathrm{OR}=1.01,95 \% \mathrm{Cl}: 1.00-1.01, p<0.001)$ and the time between anthracycline completion and trastuzumab initiation $(\mathrm{OR}=0.99,95 \% \mathrm{Cl}$ : 0.99-100, $p=0.035$ ). Also LVEF at baseline $(\mathrm{OR}=0.86,95 \% \mathrm{Cl}: 0.8-0.93, p=0.0001)$ and the LVEF drop after first three months of trastuzumab treatment $(\mathrm{OR}=1.14,95 \% \mathrm{Cl}$ : 1.07-1.21, $p<0.0001)$ were significantly associated with a significant cardiotoxic effect (Table III).

In a multivariate regression analysis the cumulative dose of anthracycline $(\mathrm{OR}=1.01,95 \% \mathrm{Cl}$ : 1.00-1.01, $p=0.002$ ) and LVEF at baseline $(\mathrm{OR}=0.83,95 \% \mathrm{Cl}: 0.70-0.99, p=0.0344)$ were independent predictors of a cardiotoxic effect.

\section{Discussion}

In randomised clinical studies trastuzumab in an adjuvant setting in women with HER-2 positive breast cancer reduced relapse of the disease by $50 \%$ and overall mortality by $30 \%$ in the 3-year follow-up [9, 24, 25]. The benefits of adjuvant 
Table III. Associations between dependent variables (significant cardiotoxicity vs. no significant cardiotoxicity) and group of independent variables in univariate logistic regression

\begin{tabular}{|c|c|c|c|c|}
\hline Independent covariates & OR & $-95 \% \mathrm{Cl}$ & $+95 \% \mathrm{Cl}$ & $P$-value \\
\hline Age & 1.02 & 0.99 & 1.06 & 0.197 \\
\hline BMI & 1.02 & 0.95 & 1.09 & 0.564 \\
\hline Hypertension & 1.38 & 0.72 & 2.64 & 0.328 \\
\hline Diabetes mellitus & 0.96 & 0.26 & 3.52 & 0.952 \\
\hline Smoking & 0.96 & 0.75 & 1.24 & 0.771 \\
\hline Hypercholesterolaemia & 1.03 & 0.53 & 2.01 & 0.931 \\
\hline Sedentary life style & 0.38 & 0.11 & 1.30 & 0.122 \\
\hline Overweight $(\leq 25 \mathrm{BMI}<30)$ & 1.13 & 0.57 & 1.78 & 0.243 \\
\hline Obesity (BMI $\geq 30$ ) & 1.35 & 0.66 & 2.78 & 0.411 \\
\hline Depression & 1.35 & 0.54 & 3.40 & 0.524 \\
\hline Positive family history & 1.39 & 0.72 & 2.68 & 0.322 \\
\hline ACE-I/ARB (at baseline) & 1.07 & 0.66 & 1.99 & 0.456 \\
\hline$\beta$-blocker (at baseline) & 1.16 & 1.19 & 1.40 & 0.329 \\
\hline Doxorubicin & 1.01 & 1.00 & 1.01 & $<0.001$ \\
\hline Cyclophosphamide & 1.04 & 0.33 & 3.25 & 0.950 \\
\hline Cyclophosphamide [g] & 1.0 & 1.00 & 1.00 & 0.234 \\
\hline Docetaxel & 0.97 & 0.79 & 1.18 & 0.744 \\
\hline Docetaxel [mg] & 1.00 & 1.00 & 1.00 & 0.608 \\
\hline 5-fluorouracyl & 0.97 & 0,84 & 1,12 & 0.655 \\
\hline 5-fluorouracyl [g] & 1.00 & 1.00 & 1.00 & 0.716 \\
\hline Adjuvant radiotherapy & 0.98 & 0.86 & 1.11 & 0.723 \\
\hline Hormone therapy & 0.72 & 0.34 & 1.53 & 0.394 \\
\hline $\begin{array}{l}\text { Time anthracycline- } \\
\text { trastuzumab }\end{array}$ & 0.99 & 0.99 & 1.00 & 0.035 \\
\hline LVEF at baseline & 0.86 & 0.80 & 0.93 & 0.0001 \\
\hline LVEF drop after first 3 months & 1.14 & 1.07 & 1.21 & $<0.0001$ \\
\hline
\end{tabular}

$B M I$ - body mass index, ACE-I - angiotensin converting enzyme inhibitor, ARB - angiotensin II receptor blockers, LVEF - left ventricle ejection fraction.

treatment were also confirmed by longer observations $[25,26]$. Unfortunately, such a high efficacy of trastuzumab is at the cost of cardiotoxicity. Trastuzumab-related cardiotoxicity occurs relatively often and is a common cause of the early discontinuation/termination of trastuzumab. In our study the therapy was stopped prematurely in every fifth woman. In most cases cardiotoxicity was in the form of asymptomatic LV systolic dysfunction $(17 \%)$ or clinically overt heart failure (2.4\%). Only 3 patients developed other, non-myocardial forms of cardiac side effects (new asymptomatic LBBB in 1 patient and new negative T-waves in ECG in 2 patients). Significant cardiotoxic effects are a threat and result in the interrup- tion or premature discontinuation of trastuzumab treatment. The development of cardiotoxicity in the course of treatment is a significant challenge that can limit its efficacy and may lead to a poorer cancer outcome.

In the present study we found that early discontinuation/termination of adjuvant trastuzumab because of cardiotoxicity in women with breast cancer negatively influenced DFS but did not influence OS. Early termination of trastuzumab resulted in a shorter time of drug exposure, which might have been responsible for the reduction in the efficacy of the therapy. Early discontinuation was associated with higher cancer recurrence. Similar results were revealed in a retrospective chart re- 
view of 2401 patients, in whom there was nearly a three-fold increase in risk of breast cancer recurrence if they had had an interruption in the course of trastuzumab treatment (17.5\% vs. 6.3\%) [27].

Patients without a cardiotoxic effect lived without relapse of cancer significantly longer by 4.6 months as compared with those who experienced cardiotoxicity. The difference is only 4.6 months but is statistically significant. It was identified during a relatively short time of observation -2 years. If the observation had been prolonged, the benefit might have improved.

Currently, the standard recommended adjuvant trastuzumab therapy in BC should last 52 weeks [28]. All trials that have tried to demonstrate equivalent effectiveness with shorter trastuzumab treatment have been inconclusive [29, 30]. In a recent meta-analysis of 4 randomized clinical trials comparing 12-month trastuzumab therapy with shorter treatment, less than 12-month trastuzumab therapy resulted in poorer overall and cancer-free survival [31]. In an adjuvant setting in $\mathrm{BC}$ patients guidelines recommend a course of treatment that is neither shorter nor longer than 52 weeks. Longer trastuzumab treatment does not improve the outcome but increases the rate of cardiac side effects [25].

The median treatment time in the group with cardiotoxic effects in our study was significantly shorter than recommended (26.9 weeks). We found that $20.55 \%$ of patients in our observation did not receive the full course of trastuzumab treatment. Trastuzumab was prematurely terminated due to cardiotoxicity, which resulted in a shorter duration of trastuzumab treatment in Group 1. This proportion is higher than in most clinical trials. For example, $8.5 \%$ of the women had trastuzumab interrupted in the HERA trial [9]. But there are some studies with a higher rate of trastuzumab discontinuation, such as $25 \%$ in the FINCLCC-PACS 04 trial [32]. In real life observations more patients must have trastuzumab treatment withheld than in clinical trials, and this is up to $40 \%$ in elderly populations $[33,34]$. The population in our study was relatively young (median age 55 years old) and did not have significant cardiovascular problems (Table I).

Early termination of trastuzumab resulted in a shorter time of drug exposure, which might have been responsible for the reduction in the efficacy of the therapy. Specifically, early trastuzumab discontinuation might have resulted in higher cancer recurrence.

No analysed cardiovascular risk factor predisposed to cardiotoxicity in our study. However, there is a well-established association between lipid disorders, cancer and cardiovascular diseases [35-37].
The only independent predictors of trastuzumab-related cardiotoxicity in the study were a cumulative dose of anthracyclines and baseline LVEF. Both neoadjuvant and adjuvant treatment contained anthracyclines and together constituted a high risk factor of trastuzumab cardiotoxicity.

Patients who developed a significant cardiotoxic effect (Group 1) had a significantly shorter time from the completion of chemotherapy to the initiation of trastuzumab. This shorter time period from the completion of chemotherapy to the initiation of trastuzumab might be a significant factor causing the cardiotoxic effect.

This was significantly associated with trastuzumab cardiotoxicity in the univariate analysis but its presence in the multivariate analysis did not reach statistical significance.

Nevertheless, some other observations suggest that this parameter is a risk factor and a very likely predisposing condition for cardiotoxicity. In the first palliative trials, when trastuzumab was used with anthracyclines concurrently, the rate of cardiotoxicity was at an extremely high, unacceptable level (LV dysfunction was observed in $27 \%$ and overt heart failure in $16 \%$ of the patients). In the adjuvant trials, trastuzumab was administered sequentially after anthracycline-based chemotherapy, which enabled the reduction of cardiotoxicity. In adjuvant NSABP and NCCTG trials, trastuzumab treatment was started 3 weeks after completion of doxorubicin, cyclophosphamide chemotherapy, whereas in the adjuvant HERA trial the interval between anthracyclines and trastuzumab was around 7 weeks. The incidence of trastuzumab related cardiotoxicity was lower in the HERA trial, which was explained by the longer time between chemotherapy completion and trastuzumab initiation. HER-2 receptor expression may be transiently upregulated by a compensatory mechanism following cardiac stress in a similar way as after exposure to anthracyclines. Peak HER-2 expression during or shortly after anthracycline treatment predisposes cardiomyocytes to trastuzumab-related cardiotoxicity. Concurrent trastuzumab may then do more harm to the heart compared to sequential administration. However, in the HERA study pre-trastuzumab LVEF values were higher and the doses of cardiotoxic epirubicin were lower than in the NSABP and NCCTG trials $[25,26]$. These two facts may also explain the lower rate of trastuzumab cardiotoxicity in the HERA trial. Alternatively, the long pause between chemotherapy and trastuzumab may impair its efficacy [38].

In our study early discontinuation/termination of trastuzumab treatment did not affect OS. Opposite observations have been made in some other studies, in which OS was reduced in patients who 
did not receive the full trastuzumab course $[33,39]$. The lack of association between cardiotoxicityrelated early termination of trastuzumab and OS in our study may be explained by (1) the short follow-up (median observation time -2 years), (2) the small number of patients, (3) the low mortality of women with non-metastatic breast cancer, (4) after having been diagnosed with a relapse of the disease, patients were intensively treated according to the guidelines, which improved their survival, (5) the relatively young age of participants (median age $=55$ ).

In all fatal cases in our observation cancer was reported as the cause of death. This is why the form of cardiotoxicity and its nature did not influence mortality (OS) during the period of observation. Cardiovascular causes of deaths in breast cancer survivors begin to dominate after 9 years from cancer diagnosis [40]. Twenty-nine women died (11.5\% of the study population) and in 8 of them trastuzumab was interrupted due to significant cardiotoxicity. Others had a recurrence of cancer during trastuzumab treatment.

Trastuzumab cardiotoxicity has been regarded as benign and reversible in most cases. Most patients who develop cardiac dysfunction experience an improvement after discontinuation of trastuzumab and implementation of standard treatment for heart failure [41]. There is more uncertainty about long-term heart complications. There have been some observations, mainly retrospective $[13,14,42]$, casting doubts on the reversibility of trastuzumab-related cardiotoxicity and suggesting that years after the completion of trastuzumab treatment cardiotoxicity may develop or persist $[13,34]$. One retrospective study showed that 5 years after the completion of breast cancer treatment $12 \%$ of women who had received trastuzumab alone and over $20 \%$ who had received trastuzumab following anthracyclines were diagnosed with heart failure or cardiomyopathy [13] In another retrospective analysis from Denmark, 5 years after trastuzumab completion the risk of heart failure development among patients treated with chemotherapy-plus-trastuzumab compared with those treated with chemotherapy alone increased two-fold [27]. In one prospective observation heart remodelling (left ventricle and left atrium enlargement) was found in a substantial number of women 6 months after the completion of BC chemotherapy consisting of anthracyclines and trastuzumab [21].

Trastuzumab cardiotoxicity may not be as innocuous as it has been regarded. Prolonged inhibition of HER-2 signalling pathways by trastuzumab may lead in some unfavourable conditions to irreversible changes in myocardium structure and function, which after many years may result in remodelling and finally in heart failure. A higher risk of heart failure development in BC survivors years after successful treatment with trastuzumab has been observed in a few retrospective analyses [21, 43].

The impact of cardiovascular risk factors on early trastuzumab-related heart damage (cardiotoxicity) is not fully understood and various observations sometimes provide conflicting conclusions. In our observation the cumulative dose of anthracycline and LVEF at baseline were independent predictors of significant trastuzumab cardiotoxicity, while none of the analysed cardiovascular factors had a substantial influence on the development of cardiotoxicity.

The molecular mechanisms of trastuzumabinduced cardiotoxicity are still not completely understood. Experimental studies on mice revealed that trastuzumab significantly altered the expression of myocardial genes for DNA repair, which was associated with ultrastructural alterations in cardiomyocytes, and also promoted oxidative stress and apoptosis of myocardium and elevated serum levels of cardiac troponin-I (cTnl) and cardiac myosin light chain-1 (cMLC1). The cardiac myofibers were disconnected and damaged, and also appeared stretched, with significantly reduced thickness. This may reflect potential inhibition of their contractile function. This suggests that trastuzumab might produce a lasting effect on myocyte structure, thus questioning the reversibility concept of trastuzumab-induced cardiotoxicity [44].

From the clinicians' perspective it is very important to make the best decision when to stop potentially lifesaving treatment. The significant drop of LVEF and clinical symptoms are two widely accepted equivalents of cardiotoxicity that influence clinical decisions. If they occur, the interruption of trastuzumab treatment is recommended. Biomarkers such as cardiac troponins and natriuretic peptides as well as some other imaging techniques such as tissue Doppler (TDI) or global longitudinal strain (GLS) are also recommended to be used for cardiotoxicity monitoring. However, biomarkers, TDI and GLS do not provide data that are relevant to the decision to interrupt trastuzumab treatment and in our opinion they were not necessary for the purpose of the study. We used a widely accepted definition of trastuzumab cardiotoxicity based on 2D echocardiography LVEF measurement and symptoms of heart failure.

The criteria for withholding trastuzumab recommended by oncology/cardiology societies are not based on evidence-based data. Unjustified withdrawal of trastuzumab treatment may negatively impact the long-term outcome, increasing the risk of cancer recurrence and mortality on the one hand. On the other hand, however, the un- 
interrupted continuation of trastuzumab despite cardiotoxic effects may result in cardiomyopathy and heart failure in the future. Trastuzumab treatment should not be terminated too early and unnecessarily in terms of oncology outcomes and not too late in terms of long-term cardiovascular consequences. The risk of early discontinuation of trastuzumab with respect to cardiac safety must be weighed against the potential loss of an anticancer effect, which may result in an increased risk of breast cancer recurrence and death [15.]. Data concerning the influence of early trastuzumab discontinuation on long-term outcomes and on the hearts of women with HER-2 positive BC are scarce in the scientific literature. Long-term prospective observations with heart morphology and function monitoring are needed.

The authors are aware of the limitations of the present study. The observation was only performed in one centre. The follow-up is too short (median follow-up = 25.7 months). The number of women with cardiotoxic effects (52) is too small, resulting from the small sample size (253 participants). The sample is relatively young and therefore may not represent common clinical practice ( $55 \pm 10$ years of age). The role of therapies preventing/treating trastuzumab-related cardiotoxicity (ACE-I/ARB; $\beta$-blockers) was not analysed because of the small number of patients. The only method for monitoring heart safety in the study was 2D echocardiography, which has many limitations. However, this is the most widely used and recommended technique to monitor cardiac safety during oncology treatment. It is readily available and not a very expensive method to image the heart. However, despite 3D echocardiography being the most reproducible technique for LVEF and LV volume measurements, it is not so widespread in clinical practice because of low availability.

The main strength of our study is its prospective design. According to the authors' knowledge, there are no studies assessing the influence of trastuzumab-related cardiotoxicity on OS and DFS prospectively. All available studies on this issue are retrospective analyses. The results of our study and of others previously published support the strategy to assess the risk benefit ratio thoroughly in each individual before making the decision to stop or to continue trastuzumab treatment in the case of cardiotoxicity.

In conclusion, cardiotoxicity associated with trastuzumab that results in early treatment discontinuation negatively influences DFS but seems not to influence OS in patients treated for unilateral, non-metastatic breast cancer in an adjuvant setting. Further in-depth observations are needed to confirm these conclusions.
Trastuzumab-related cardiotoxicity occurs relatively often and is a common cause of the early termination of trastuzumab treatment. Asymptomatic LV dysfunction is the most frequent form of trastuzumab-related cardiac side effects. Symptomatic heart failure and ECG abnormalities occur less often.

The cumulative doses of anthracycline and LVEF at baseline are independent predictors of significant trastuzumab cardiotoxicity.

\section{Conflict of interest}

The authors declare no conflict of interest.

\section{References}

1. Slamon DJ, Godolphin W, Jones LA, et al. Studies of the HER-2/neu proto-oncogene in human breast and ovarian cancer. Science 1989; 244: 707-12.

2. Slamon DJ, Clark GM, Wong SG, Levin WJ, Ullrich A, McGuire WL. Human breast cancer: correlation of relapse and survival with amplification of the HER-2/neu oncogene. Science 1987; 235: 177-82.

3. Slamon D, Eiermann W, Robert N, et al. On behalf of the Breast Cancer International Research Group. Adjuvant trastuzumab in HER2-positive breast cancer. N Engl J Med 2011; 365: 1273-83.

4. Moja L, Tagliabue L, Balduzzi S, et al. Trastuzumab containing regimens for early breast cancer. Cochrane Database Syst Rev 2012; 4: CD006243.

5. de Azambuja E, Procter MJ, van Veldhuisen DJ, et al. Trastuzumab-associated cardiac events at 8 years of median follow-up in the Herceptin Adjuvant trial (BIG1-01). J Clin Oncol 2014; 32: 2159-65.

6. Perez EA, Suman VJ, Davidson NE, et al. Cardiac safety analysis of doxorubicin and cyclophosphamide followed by paclitaxel with or without trastuzumab in the North Central Cancer Treatment Group N9831 adjuvant breast cancer trial. J Clin Oncol 2008; 26: 1231-8.

7. Romond EH, Jeong JH, Rastogi P, et al. Seven-year follow-up assessment of cardiac function in NSABP B-31, a randomized trial comparing doxorubicin and cyclophosphamide followed by paclitaxel (ACP) with ACP plus trastuzumab as adjuvant therapy for patients with node-positive, human epidermal growth factor receptor 2-positive breast cancer. J Clin Oncol 2012; 30: 3792-9.

8. Tan-Chiu E, Yothers G, Romond E, et al. Assessment of cardiac dysfunction in a randomized trial comparing doxorubicin and cyclophosphamide followed by paclitaxel, with or without trastuzumab as adjuvant therapy in node-positive, human epidermal growth factor receptor 2-overexpressingbreast cancer: NSABP B-31. J Clin Oncol 2005; 23: 7811-9.

9. Piccart-Gebhart MJ, Procter M, Leyland-Jones B, et al. Trastuzumab after adjuvant chemotherapy in HER2-positivebreast cancer. N Engl J Med 2005; 353: 1659-72.

10. Tolaney SM, Barry WT, Dang CT, et al. Adjuvant paclitaxel and trastuzumab for node-negative, HER2-positivebreast cancer. N Engl J Med 2015; 372: 134-41.

11. Jones SE, Collea R, Paul D, et al. Adjuvant docetaxel and cyclophosphamide plus trastuzumab inpatients with HER2-amplified early stage breast cancer: a single group, open-label, phase 2 study. Lancet Oncol 2013; 14: 1121-8. 
12. Telli ML, Hunt SA, Carlson RW, Guardino AE. Trastuzumab-related cardiotoxicity: calling into question the concept of reversibility. J Clin Oncol 2007; 25: 3525-33.

13. Bowles EJ, Wellman R, Feigelson HS, et al.; Pharmacovigilance Study Team. Risk of heart failure in breast cancer patients after anthracycline and trastuzumab treatment: a retrospective cohort study. J Natl Cancer Inst 2012; 104: 1293-305.

14. Banke A, Fosbøl EL, Ewertz M, et al. Long-term risk of heart failure in breast cancer patients after adjuvant chemotherapy with or without trastuzumab. JACC Heart Fail 2019; 7: 217-24.

15. Barron CC, Tyagi NK, Alhussein MM, et al. Adjuvant trastuzumab therapy: can we balance efficacy and safety? Oncologist 2019; 24: 1405-9.

16. Mackey JR, Clemons M, Cote MA, et al. Cardiac management adjuvant trastuzumab therapy: recommendation of the Canadian Working Group. Current Oncol 2008; 15: 24-35.

17. Jones AL, Barlow M, Barrett-Lee PJ, et al. Management of cardiac health in trastuzumab treated patients with breast cancer: updated United Kingdom National Cancer Research Institute recommendations for monitoring. Br J Cancer 2009; 100: 684-92.

18. Swedberg K, Cleland J, Dargie H, et al. Guidelines for the diagnosis and treatment of chronic heart failure: full text (update 2005). The task force for the diagnosis and treatment of CHF of the European Society of Cardiology. Eur Heart J 2005; 26: 1115-40.

19. Douglas PS, DeCara JM, Devereux RB, et al. Echocardiographic imaging in clinical trials: American Society of Echocardiography Standards for echocardiography core laboratories: endorsed by the American College of Cardiology Foundation. J Am Soc Echocardiogr 2009; 22: 755-65.

20. Guarneri V, Lenihan DJ, Valero V, et al. Long-term cardiac tolerability of trastuzumab in metastatic breast cancer: the M.D. Anderson Cancer Center experience. J Clin Oncol 2006; 24: 4107-15.

21. Piotrowski G, Gawor R, Bourge RC, et al. Heart remodelling induced by adjuvant trastuzumab-containing chemotherapy for breast cancer overexpressing human epidermal growth factor receptor type 2: a prospective study. Pharmacol Res 2013; 78: 41-8.

22. Tan-Chiu E, Yothers G, Romond E, et al. Assessment of cardiac dysfunction in a randomized trial comparing doxorubicin and cyclophosphamide followed by paclitaxel, with or without trastuzumab as adjuvant therapy in node-positive, human epidermal growth factor receptor2-overexpressing breast cancer: NSABP B-31. J Clin Oncol 2005; 23: 7811-9.

23. Romond EH, Perez EA, Bryant J, et al. Trastuzumab plus adjuvant chemotherapy for operable HER2-positive breast cancer. N Engl J Med 2005; 353: 1673-84.

24. Russell SD, Blackwell KL, Lawrence J, et al. Independent adjudication of symptomatic heart failure with the use of doxorubicin and cyclophosphamide followed by trastuzumab adjuvant therapy: a combined review of cardiac data from the National Surgical Adjuvant breast and Bowel Project B-31 and the North Central Cancer Treatment Group N9831 clinical trials. J Clin Oncol 2010; 28: 3416-21.

25. Cameron D, Piccart-Gebhart MJ, Gelber RD, et al. Herceptin Adjuvant (HERA) Trial Study Team. 11 years' follow-up of trastuzumab after adjuvant chemotherapy in HER2-positive early breast cancer: final analysis of the HERceptin Adjuvant (HERA) trial. Lancet 2017; 389: 1195-205.
26. Perez EA, Romond EH, Suman VJ, et al. Four-year follow-up of trastuzumab plus adjuvant chemotherapy for operable human epidermal growth factor receptor 2-positive breast cancer: joint analysis of data from NCCTG N9831 and NSABP B-31. J Clin Oncol 2011; 29: 3366-73.

27. Gibson J, Yao RJ, Davis $M$ et al. The impact of mild left ventricular dysfunction on trastuzumab use and oncologic outcomes in early stage breast cancer therapy. J Clin Oncol 2018; 35: e18148a.

28. Senkus E, Kyriakides S, Ohno S, et al.; ESMO Guidelines Committee. Primary breast cancer: ESMO Clinical Practice Guidelines for diagnosis, treatment and follow-up. Ann Oncol 2015; 26 Suppl 5: v8-30.

29. Earl HM, Hiller L, Vallier AL, et al. on behalf of the Persephone Steering Committee and Trial Investigators. 6 versus 12 months of adjuvant trastuzumab for HER2-positive early breast cancer (Persephone): 4-year disease-free survival results of a randomised phase 3 non-inferiority trial. Lancet 2019; 393: 2599-612.

30. Pivot X, Romieu G, Debled $M$, et al. PHARE trial investigators. 6 months versus 12 months of adjuvant trastuzumab in early breast cancer (PHARE): final analysis of a multicentre, open-label, phase 3 randomised trial. Lancet 2019; 393: 2591-8.

31. Gyawali B, Niraula S. Duration of adjuvant trastuzumab in HER2 positive breast cancer: overall and disease free survival results from meta-analyses of randomized controlled trials. Cancer Treat Rev 2017; 60: 18-23.

32. Spielmann $M$, Roche $H$, Delozier $T$, et al. Trastuzumab for patients with axillary-node-positive breast cancer: results of the FNCLCC-PACS 04 trial. J Clin Oncol 2009; 27: 6129-34.

33. Wang SY, Long JB, Hurria A, et al. Cardiovascular events, early discontinuation of trastuzumab, and their impact on survival. Breast Cancer Res Treat 2014; 146: 411-9.

34. Chen J, Long JB, Hurria A, Owusu C, Steingart RM, Gross CP. Incidence of heart failure or cardiomyopathy after adjuvant trastuzumab therapy for breast cancer. J Am Coll Cardiol 2012; 60: 2504-12.

35. Penson P, Long DL, Howard G, et al. Associations between cardiovascular disease, cancer, and very low high-density lipoprotein cholesterol in the REasons for Geographical and Racial Differences in Stroke (REGARDS) study. Cardiovasc Res 2019; 115: 204-12.

36. Ganjali S, Ricciuti B, Pirro M, et al. High-density lipoprotein components and functionality in cancer: state-ofthe-art. Trends Endocrinol Metab 2019; 30: 12-24.

37. Mazidi M, Katsiki N, Mikhailidis DP, et al. Apolipoprotein B/ apolipoprotein A-I ratio is a better predictor of cancer mortality compared with C-reactive protein: results from two multi-ethnic US populations. J Clin Med 2020; 9: 170 .

38. Petrelli F, Barni S. Meta-analysis of concomitant compared to sequential adjuvant trastuzumab in breast cancer: the sooner the better. Med Oncol 2012; 29: 50310.

39. Gong IY, Verma S, Yan AT, et al. Long-term cardiovascular outcomes and overall survival of early-stage breast cancer patients with early discontinuation of trastuzumab: a population-based study. Breast Cancer Res Treat 2016; 157: 535-44

40. Patnaik JL, Byers T, DiGuiseppi C, et al. Cardiovascular disease competes with breast cancer as the leading cause of death for older females diagnosed with breast cancer: a retrospective cohort study. Breast Cancer Res 2011; 13: R64. 
41. Seidman A, Hudis C, Pierri MK, et al. Cardiac dysfunction in the trastuzumab clinical trials experience. J Clin Oncol 2002; 20: 1215-21.

42. Bowles EA, Wellman R, Delate T, et al. C-B2-01: Cardiotoxic chemotherapy is associated with increased heart failure risk among women with breast cancer in the cancer research network. Clin Med Res 2011; 9: 148.

43. Du XL, Xia R, Burau K, Liu CC. Cardiac risk associated with the receipt of anthracycline and trastuzumab in a large nationwide cohort of older women with breast cancer, 1998-2005. Med Oncol 2011; 28 Suppl 1: S80-90.

44. ElZarrad MK, Mukhopadhyay P, Mohan N, et al. Trastuzumab alters the expression of genes essential for cardiac function and induces ultrastructural changes of cardiomyocytes in mice. PLoS One 2013; 8: e79543. 